\title{
3D Medical image compression using the quincunx wavelet coupled with SPIHT
}

\author{
Benlabbes Haouari \\ Department of exact sciences, Higher Normal School of Bechar, Algeria
}

\begin{tabular}{l}
\hline \hline Article Info \\
\hline Article history: \\
Received Aug 1, 2019 \\
Revised Nov 2, 2019 \\
Accepted Nov 16, 2019 \\
\hline
\end{tabular}

\section{Keywords:}

3D Medical image

Compression

Quincunx

SPIHT

\begin{abstract}
Medical imaging is a growing field due to the development of digital technologies that produce $3 \mathrm{D}$ and even $4 \mathrm{D}$ data. The counterpart to the resolution offered by these voluminal images resides in the amount of gigantic data, hence the need for compression. This article presents a new coding scheme dedicated to 3D medical images. The originality of our approach lies in the application of the Quinqunx wavelet transform coupled with the SPIHT encoder on a database of medical images. This approach achieves much higher compression rates, while maintaining a very acceptable visual quality.
\end{abstract}

Copyright $@ 2020$ Institute of Advanced Engineering and Science. All rights reserved.

\section{Corresponding Author:}

Benlabbes Haouari,

Department of exact sciences,

Higher Normal School of Bechar, Algeria.

Email: haouari.benlabbes@ens.univ-bechar.dz

\section{INTRODUCTION}

Medical imaging has made very significant progress in recent years with the development of techniques that produce 3D data more and more accurate but in return for more voluminous. Some of these images are intrinsically volumic while others correspond to a succession of 2D images (slices images) [1-5]. The increasing increase in storage capacity provides a partial answer to this problem but remains insufficient. In addition to the issue of archiving, the transmission of these images on bandwidths by nature is also a problem.

Therefore, the compression of volume medical images cannot be avoided: it involves reducing the number of bits necessary for the faithful representation of the original image and to access only the required information, thus facilitating the transmission and allowing a remote access to data.

In this paper we present a 3D medical image compression approach based on the Quinqunx wavelet transform coupled with the SPIHT encoder on a medical image database. This is recognized as a decorrelating transformation very effective for this type of images [6]. This provides a significant improvement in the rate-distortion compromise. The numerical and visual results produced by the coupling of Quincunx and SPIHT on the images are very promising.

\section{RESEARCH APPROACH}

The general scheme of our compression approach is shown in Figure 1. The 3D image contains $\mathrm{z}$ slices [6-8] (one slice represents a 2D image ( $x, y)$ ), in the compression phase, in the first step, a quincun $x$ transformation is applied on the slice $\mathrm{i}(\mathrm{i}=1$ to $\mathrm{z}$ ). Second, we enter the result of the transformation in the progressive encoder SPIHT (set of partitioning in hierarchical trees) is a compression algorithm for the compression of wavelet transform coefficients. It was introduced by Amir Said and William A. Pearlman in 
1996 [9]. More concretely, SPIHT progressively transforms these coefficients into a bit stream [10-11]. This stream can be cut anywhere. During decoding, the coefficients are more and more refined. Now we have a compressed image.

The decompression step reverses the compression process until a reconstructed image is obtained. This process is repeated until the last slice ( $\mathrm{z}$ slice) of the 3D image. Finally, we get a 3D image after grouping all slices $(1 \ldots \mathrm{z})$.

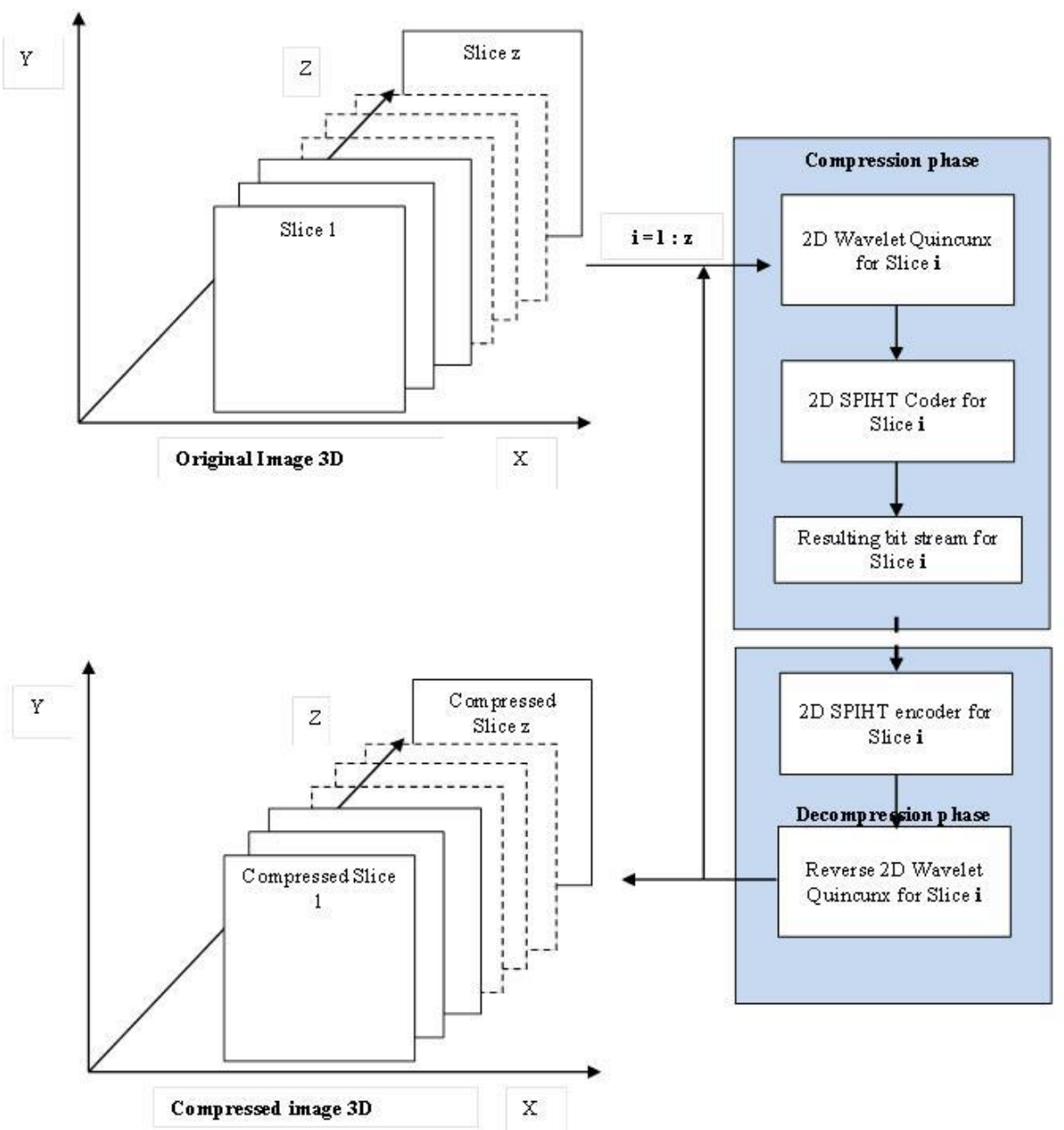

Figure 1. Proposed overall scheme of compression

\section{QUINCUNX WAVELET TRANSFORM}

The purpose of the quincunx wavelet is to improve the boundaries of separable wavelets. To overcome these limitations, wavelets based on staggered sampling suitable for analyzing the entire image and not the rows and columns. The quincunx method used in this article is described in [12-13].

\section{SPIHT ENCODER}

The SPIHT algorithm [14-17] uses the principles mentioned in EZW while proposing to recursively partition the coefficient trees. Thus, where EZW coded an isolated insignificant coefficient ('Z'), SPIHT performs a recursive partitioning of the tree so as to determine the position of the significant coefficients in the progeny of the considered coefficient. The significant coefficients are coded in a manner similar to EZW: their sign is sent as soon as they are identified as significant and they are added to the list of coefficients to be refined. This algorithm also works by bit planes. It offers outstanding performance. The bits sent during the signifiance pass correspond to the program executed at the encoder during the execution of the algorithm of 
classification into significant and insignificant coefficients. By following the same program, the decoder remains synchronous with the decisions of the encoder and finds the same classification. This algorithm is based on the management of three lists, significant coefficients (LSP), insignificant coefficients (LIP) and insignificant sets (LIS) [18-20]. With a significance threshold divided by two at each iteration, and whose initial value is transmitted to the decoder, the algorithm proceeds as follows. The list of significant coefficients is initially empty, while the list of insignificant coefficients contains the roots of each tree (coefficients of the low band) and the list of insignificant sets contains all the descendants of each tree. This initial partition is segmented recursively by means of two rules. If a set of descendants of a node is significant, it is separated into four direct child coefficients of this node, and all the other descendants [18-23].

Direct wires are added to the LIP or LSP depending on their significance. If at least one element of all other descendants is significant, this set is separated into four insignificant sets added to the LIS. Treating the coefficients in groups of four allows efficient entropy coding later. As in EZW, the refinement pass consists of progressively coding the least significant bits of the significant coefficients. Since the coefficients are coded in groups of four, it is interesting to treat them globally in order to exploit entropy of order greater than 1. The coefficients can only pass from the insignificant state to the signifying state; the size of the necessary alphabet to represent these changes varies according to the number of coefficients already signifying in the group [18-23].

\section{ASSESSMENT MEASURES}

Peak Signal to Noise Ratio (PSNR) is a metric for calculating degradation in a digital image, especially in image compression.

The PSNR between two tranches (original slice and their compressed slice) is calculated by the following formula:

$$
\operatorname{PSNR}_{d B}=\log _{10}\left(255^{2} / \frac{1}{x^{*} y} \sum_{i=1}^{x} \sum_{j=1}^{y} \mid \text { Slice }_{i, j}-\text { Slice }\left._{i, j}\right|^{2}\right)
$$

The average PSNR between the original 3D image and their compression result is calculated by the following formula:

$$
\operatorname{MPSNR}_{d B}=\frac{\sum_{i=1}^{z} P S N R}{z}
$$

Structural SIMilarity (SSIM), measure the visual quality of a compressed image, compared to the original image. The similarity compares the brightness, contrast and structure between each pair of vectors, the structural similarity index (SSIM) between two signals and calculated by the following formula:

$$
\operatorname{SSIM}(x, y)=l(x, y) \cdot c(x, y) \cdot s(x, y)
$$

Finally, the quality measurement can provide a map of the quality of the local image, which provides more information about the degradation of image quality.

For application, we need a single overall measure of the overall quality of the image that is given by the following formula:

$$
\operatorname{MSSIM}(\text { Slice }, \text { Slice })=\frac{1}{z} \sum_{i=1}^{z} \operatorname{SSIM}\left(\text { Slice }_{i}, \text { Slice }_{i}\right)
$$

Where Slice and Slice are the reference and degraded images, respectively, Slice $\mathrm{i}_{\mathrm{i}}$ and $\widehat{\text { Slice }}_{\mathrm{i}}$ are the contents of the images to the local window i.

$\mathrm{M}$ is the total number of local windows in the image. The MSSIM values show greater consistency with the visual quality.

The average MSSIM between the 3D original image and their compression result is calculated by the following formula: 
MMSSIM $=\frac{\sum_{i=1}^{z} \text { Slice }_{i}-\text { Slice }_{i}}{z}$

\section{EXPERIMENTAL RESULTS}

We tested the approach on 3D images "image 1,size $(256,256,108)$ " [24], "image 2, size $(256,256.5)$ " [25],"image $3(256,256.6)$ " [26] and "image 4 [27].

As shown in Figure 6 and the graphs in Figures 7 and 8 below, for a bit rate $=0.3 \mathrm{bpp}$, the results are excellent (PSNR and MSSIM), which gives reconstructed images of very good quality with a very high compression ratio. We conclude that the effectiveness of the presented algorithm for 3D medical images. Original image 1 till 5 as shown in Figures 2-5.
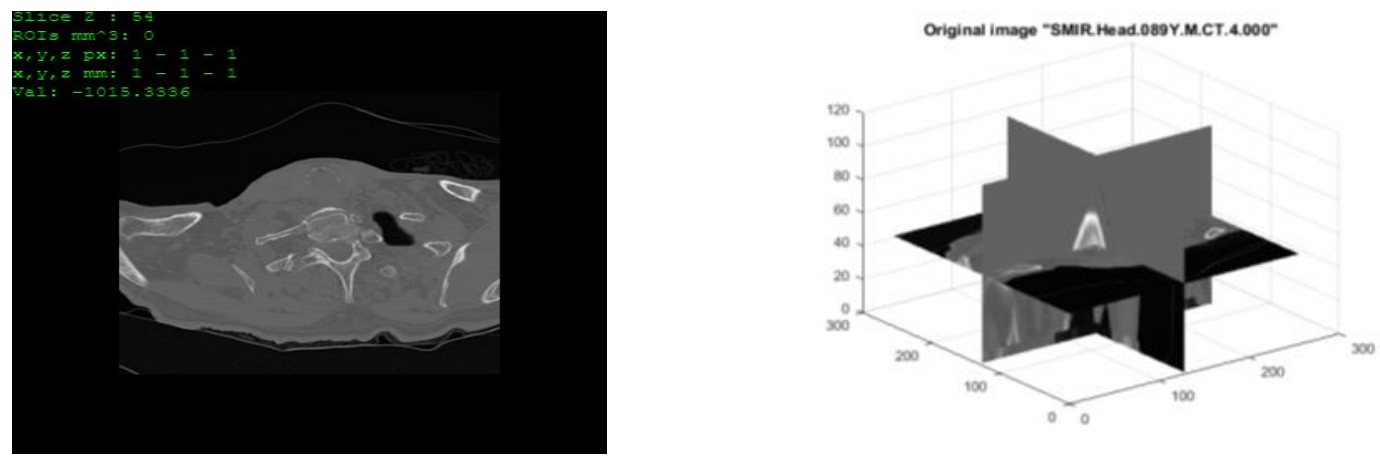

Figure 2. Original image 1
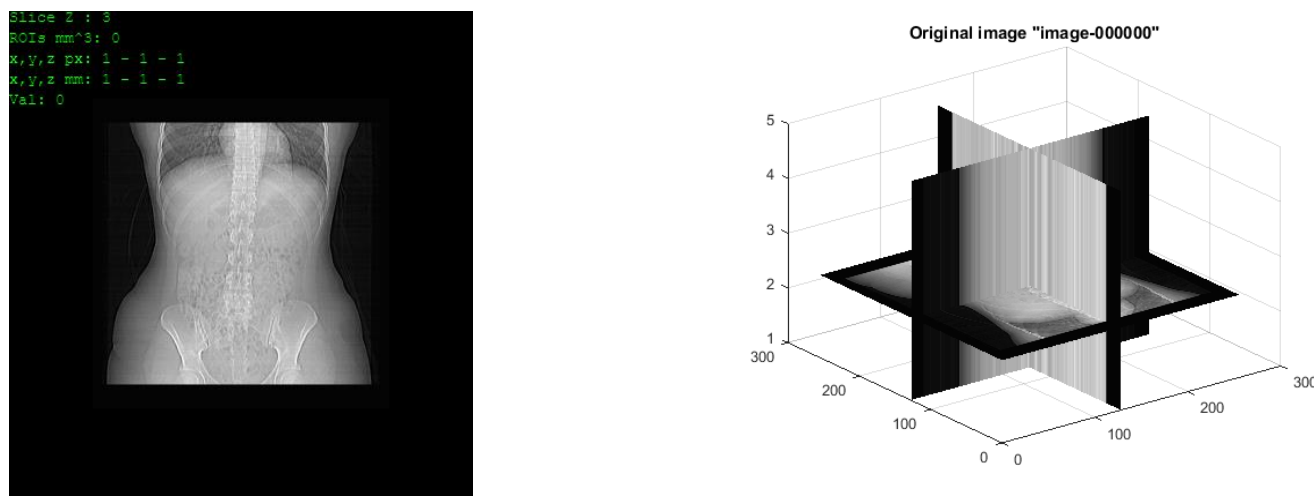

Figure 3. Original image 2
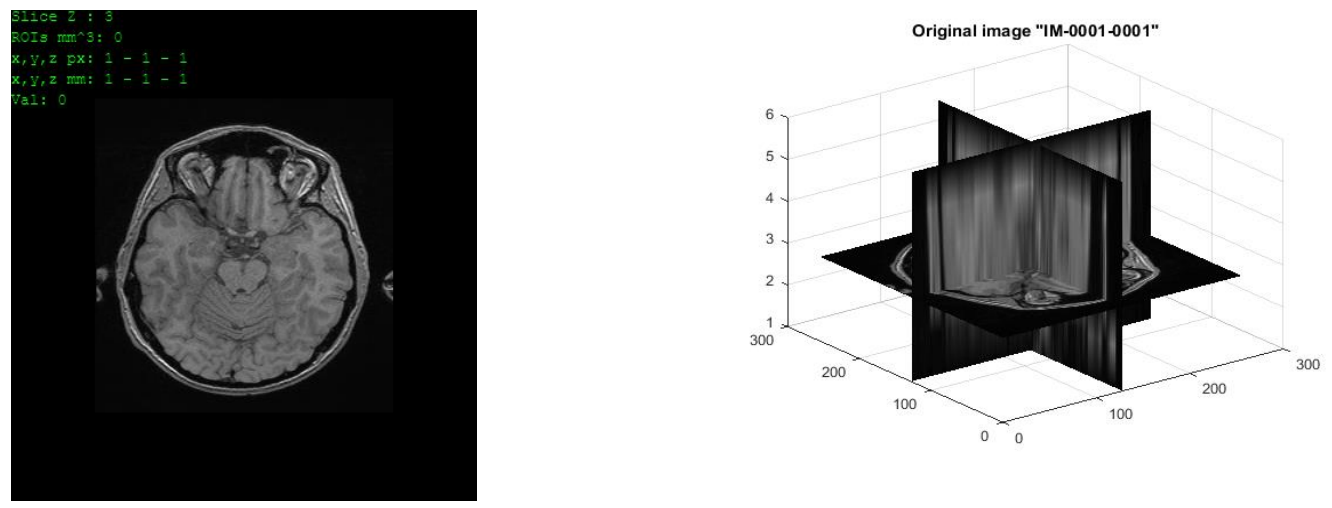

Figure 4. Original image 3 

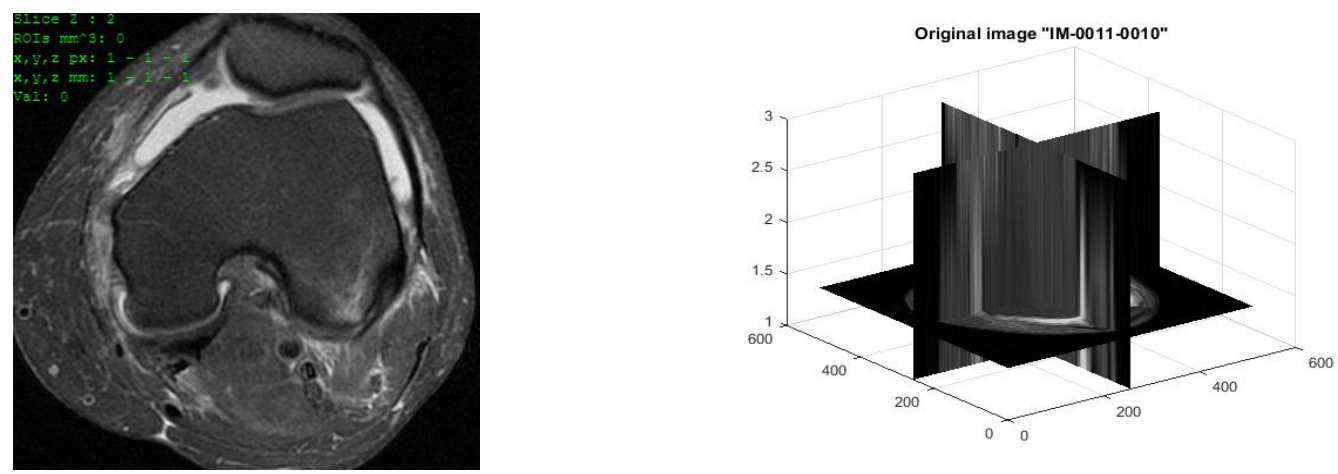

Figure 5. Original image 4
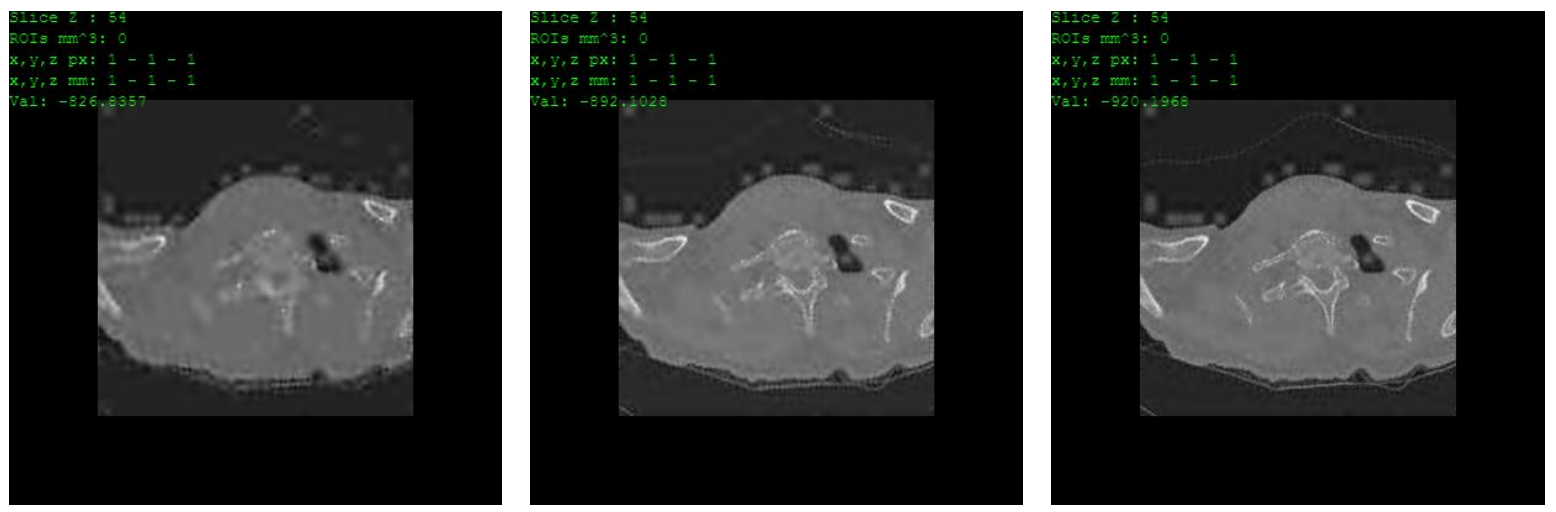

Compresued image weth bartate 0.1 bep

Compressed image weth barate 0.2 bpp

Compressed imape weth betrate es bpp

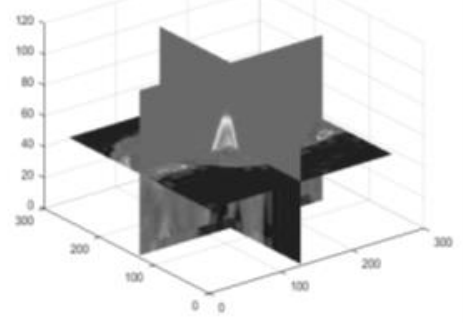

Bitrate $=0.10 \mathrm{bpp}$

Ratio compression $=98.75 \%$

MPSNR $=20.97 \mathrm{~dB}$

MMSSIM $=0.58$

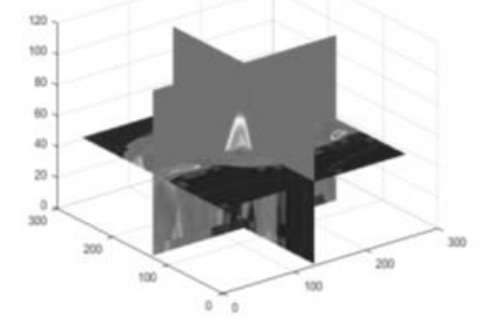

Bitrate $=0.20 \mathrm{bpp}$

Ratio compression $=97.50 \%$

MPSNR $=27.71 \mathrm{~dB}$

MMSSIM $=0.64$

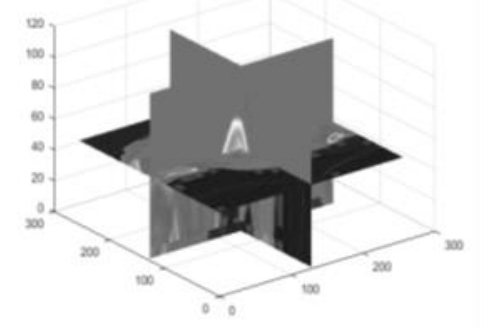

Bitrate $=0.30 \mathrm{bpp}$

Ratio compression $=96.25 \%$

MPSNR $=32.38 \mathrm{~dB}$

MMSSIM $=0.68$
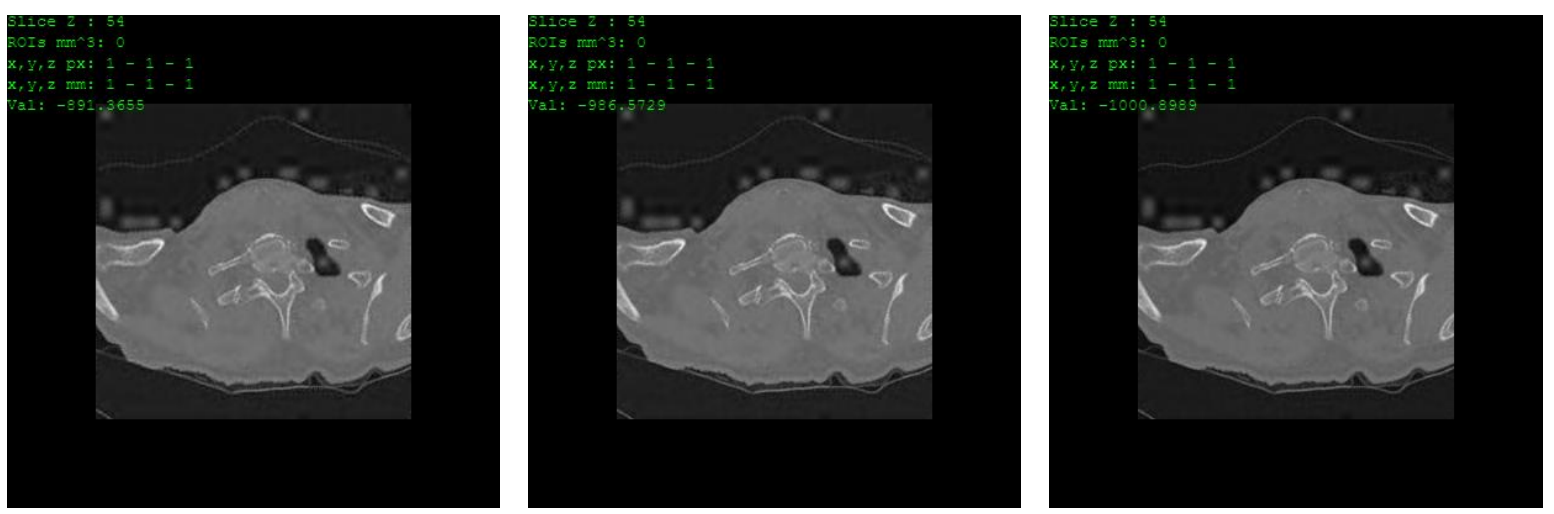


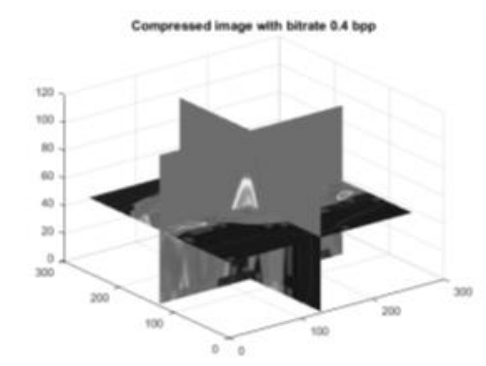

Bitrate $=0.40 \mathrm{bpp}$

Ratio compression $=95.00 \%$

MPSNR $=36.20 \mathrm{~dB}$

$\mathrm{MMSSIM}=0.72$

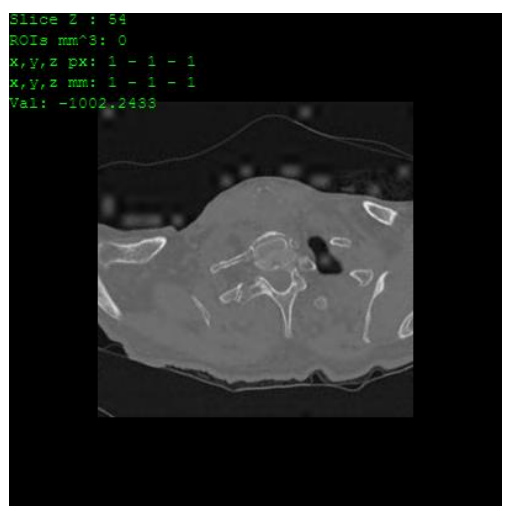

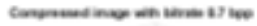

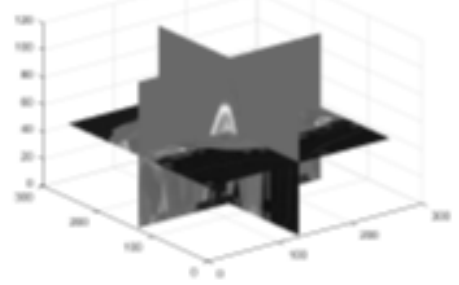

Bitrate $=0.70 \mathrm{bpp}$

Ratio compression $=91.25 \%$

MPSNR $=44.80 \mathrm{~dB}$

MMSSIM $=0.78$

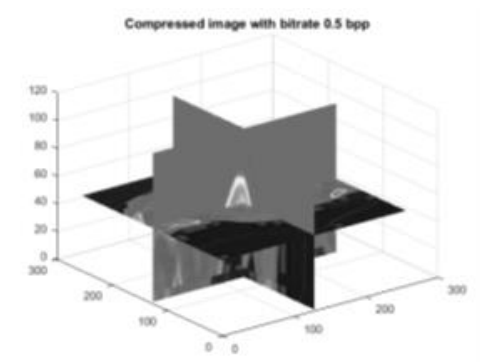

Bitrate $=0.50 \mathrm{bpp}$

Ratio compression $=93.75 \%$

MPSNR $=39.44 \mathrm{~dB}$

MMSSIM $=0.74$

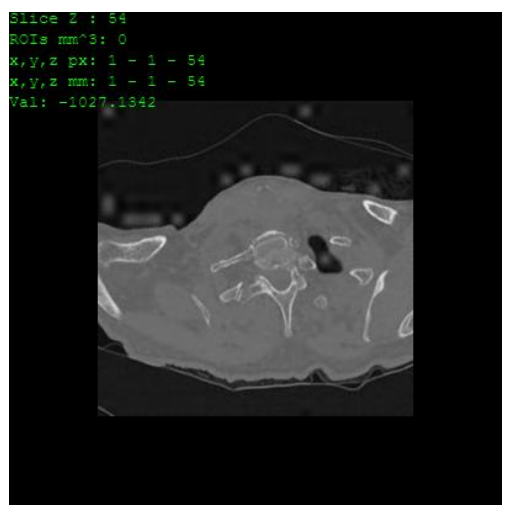

Compreeseed imase weth batrate as bop

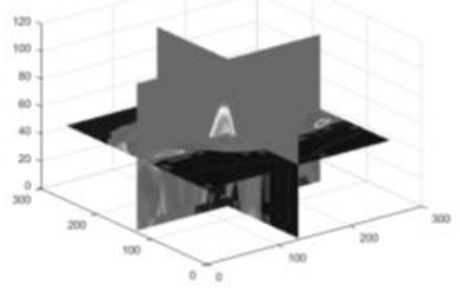

Bitrate $=0.80 \mathrm{bpp}$

Ratio compression $=90.00 \%$

MPSNR $=47.09 \mathrm{~dB}$

$\mathrm{MMSSIM}=0.80$

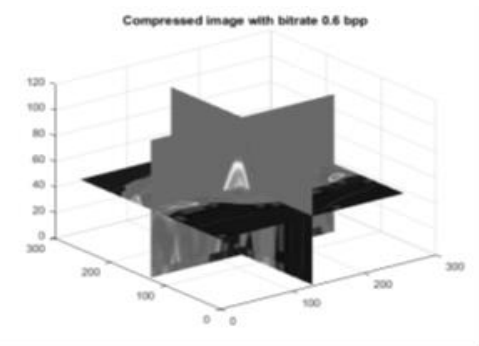

Bitrate $=0.60 \mathrm{bpp}$

Ratio compression $=92.50 \%$

MPSNR $=42.26 \mathrm{~dB}$

MMSSIM $=0.76$
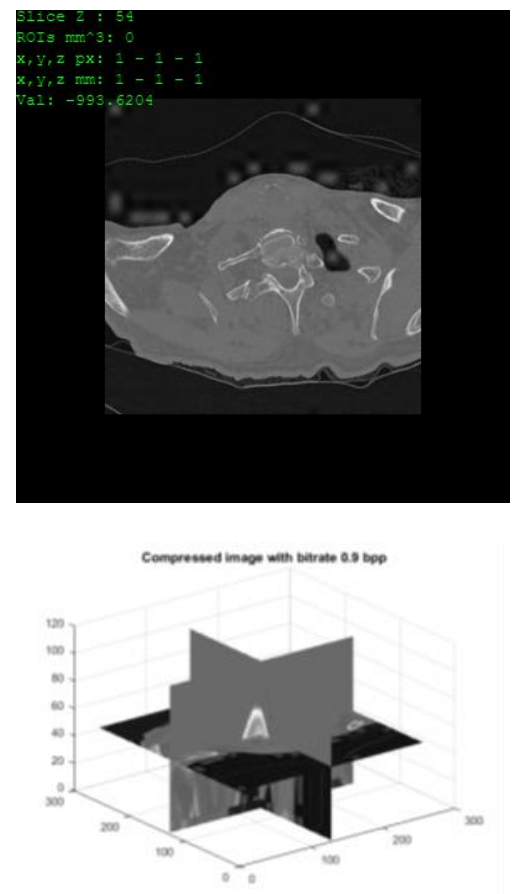

Bitrate $=0.90 \mathrm{bpp}$

Ratio compression $=88.75$

MPSNR $=49.24 \mathrm{~dB}$

MMSSIM $=0.81$
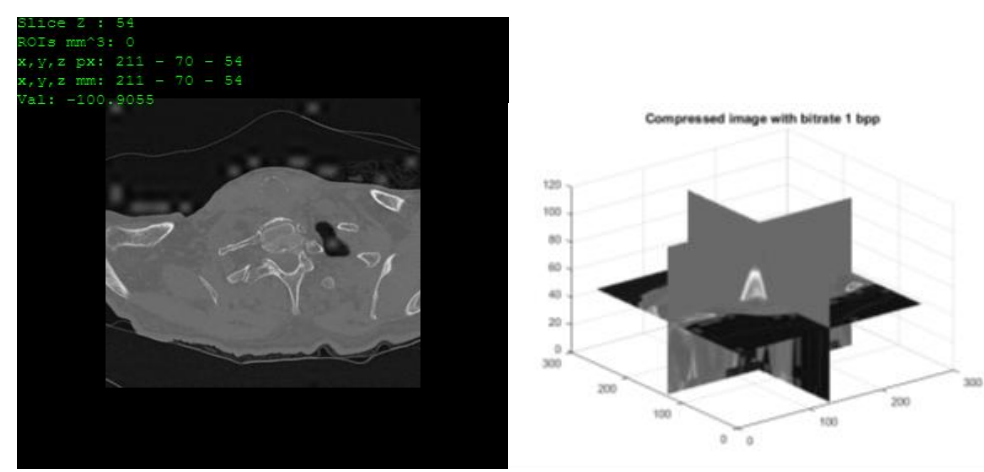

Bitrate $=1.00 \mathrm{bpp}$

Ratio compression $=87.50$

MPSNR $=51.26 \mathrm{~dB}$

MMSSIM $=0.82$

Figure 6. MPSNR and MMSSIM variation results for image 1 


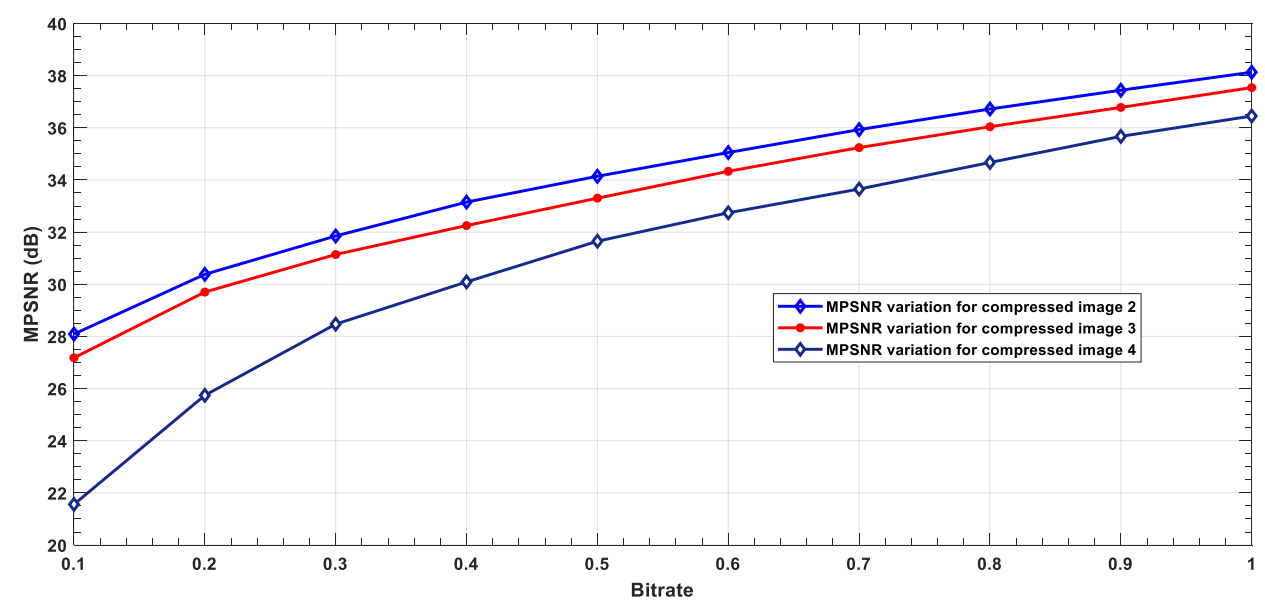

Figure 7. MPSNR variation for compressed images 2, 3 and 4

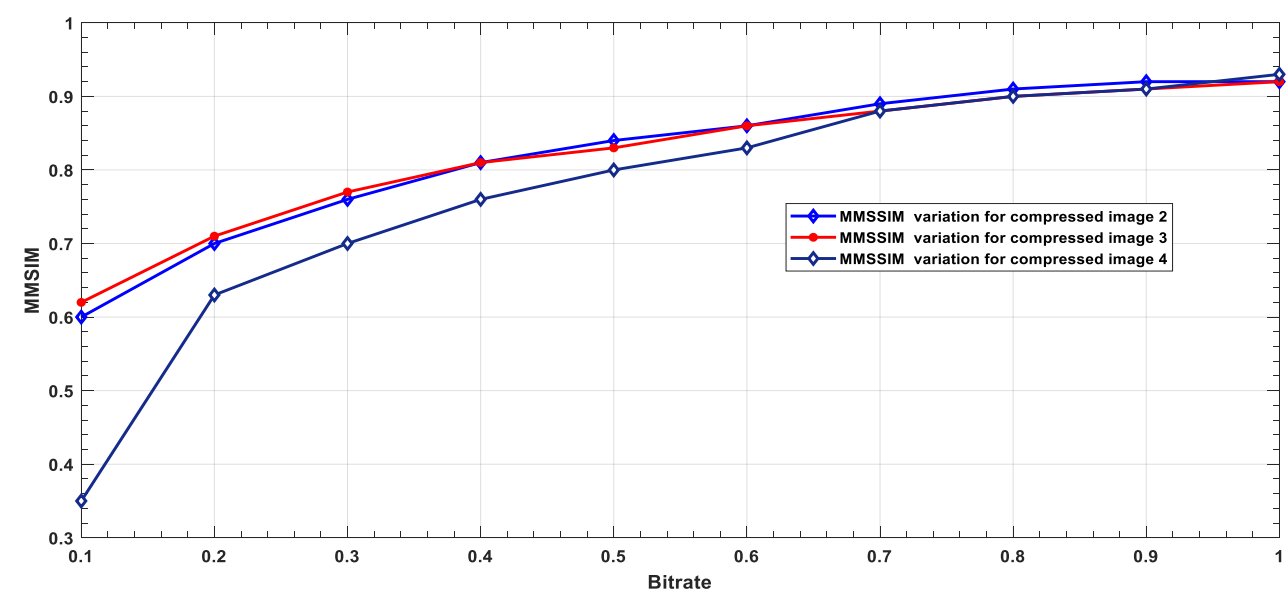

Figure 8. MMSSIM variation for compressed images 2, 3 and 4

\section{CONCLUSION}

In this paper, we proposed a 3D medical image compression approach using the quinqunx wavelet transform coupled with the SPIHT encoder. This approach has been tested on 3D medical images (2D slices), from bitrate equal $0.3 \mathrm{bpp}$, the results obtained are satisfactory in terms of PSNR and MSSIM; interesting compression rates and good quality of reconstructed images.

\section{REFERENCES}

[1] D. Ravichandran, M. G. Ahamad, and M. A. Dhivakar, "Performance analysis of three-dimensional medical image compression based on discrete wavelet transform," in 2016 22nd International Conference on Virtual System \& Multimedia (VSMM), 2016, pp. 1-8.

[2] B. Fan, "Selective Compression of Medical Images via Intelligent Segmentation and 3D-SPIHT Coding," 2018.

[3] S. Vakili and M. Khalili, "A joint 3D DWT and SPIHT based algorithm for 3D MRI image compression," in 2015 5th International Conference on Computer and Knowledge Engineering (ICCKE), 2015, pp. 36-41.

[4] P. Jangbari and D. Patel, "Review on region of interest coding techniques for medical image compression," International Journal of Computer Applications, vol. 134, pp. 1-5, 2016.

[5] N. M. Banu and S. Sujatha, "3D medical image compression: a review," Indian Journal of Science and Technology, vol. 8, p. 1, 2015.

[6] M. F. Aly and M. A. Mahmood, "3D Medical Images Compression," in Intelligent Multidimensional Data and Image Processing, ed: IGI Global, 2018, pp. 259-279.

[7] S. Liu, W. Bai, N. Zeng, and S. Wang, "A Fast Fractal Based Compression for MRI Images," IEEE Access, vol. 7, pp. 62412-62420, 2019. 
[8] S. Juliet, E. B. Rajsingh, and K. Ezra, "Projection-based medical image compression for telemedicine applications," Journal of digital imaging, vol. 28, pp. 146-159, 2015.

[9] A. Said and W. A. Pearlman, "A New, Fast, and Efficient Image Codec Based on Set Partitioning in Hierarchical Trees," IEEE Transactions on Circuits and Systems for Video Technology, vol. 6, 1996-06.

[10] Y. Habchi, M. Beladgham, H. Benlabbes, and A. T. Abdelmalik, "Hybrid DCT-DBT Algorithm for Medical Video Compression," Electrotehnica, Electronica, Automatica, vol. 64, p. 97, 2016.

[11] G. Poornima and S. P. Kumar, "Efficient H. 264 Decoder Architecture Using External Memory and Pipelining," Indonesian Journal of Electrical Engineering and Computer Science (IJEECS), vol. 12, pp. 995-1002, 2018.

[12] H. Benlabbes, K. Benahmed, M. Beladgham, A. T. Abdelmalik, and K. Younes, "A modified QWT for image transmission in WMSN: Study and Experimental," International Journal of Computer Science and Network Security, vol. 17, pp. 70-75, 2017.

[13] B. Ismahane, B. Abdesselam, A. C. E. houda, and B. Mohammed, "Modified spiht algorithm for quincunx wavelet image coding," Indonesian Journal of Electrical Engineering and Computer Science (IJEECS), vol. 16, pp. 230-242, 2019.

[14] Y.-Y. Chen, "Medical image compression using DCT-based subband decomposition and modified SPIHT data organization," International Journal of Medical Informatics, vol. 76, pp. 717-725, 2007.

[15] S.-G. MIAOU, S.-T. CHEN, and S.-N. CHAO, "Wavelet-based lossy-to-lossless medical image compression using dynamic VQ and SPIHT coding," Biomedical Engineering: Applications, Basis and Communications, vol. 15, pp. 235-242, 2003.

[16] A. Said and W. A. Pearlman, "A new, fast, and efficient image codec based on set partitioning in hierarchical trees," IEEE Transactions on circuits and systems for video technology, vol. 6, pp. 243-250, 1996.

[17] H. Al-Tameemi and T. Ibrahim, "Analyze Medical Image and Compress It by Different Algorithms," International Journal of Computer Science and Software Engineering, vol. 6, pp. 284-293, 2017.

[18] K.-h. Park and H. W. Park, "Region-of-interest coding based on set partitioning in hierarchical trees," IEEE Transactions on Circuits and Systems for Video Technology, vol. 12, pp. 106-113, 2002.

[19] X. Rong, H. Nie, W. Wang, C. Lin, and X. Yu, "SPIHT-Based Image Compression Using Optimization of LIS and LIP Encoding," JCP, vol. 13, pp. 1385-1394, 2018.

[20] C.-M. Song, B. Fu, X.-H. Wang, and M.-Z. Fu, "Wavelet Video Coding Algorithm Based on Energy Weighted Significance Probability Balancing Tree," arXiv preprint arXiv:1808.09640, 2018.

[21] K. Ravi, C. Kamargaonkar, and M. Sharma, "Comparative Study of PCA and SPIHT Methods in Medical image Compression," i-manager's Journal on Image Processing, vol. 3, p. 1, 2016.

[22] R. M. Reddy, K. Ravichandran, B. Venkatraman, and S. Suganya, "A new approach for the image compression to the medical images using PCA-SPIHT," Biomedical Research (0970-938X), 2018.

[23] S. Bajpai, H. V. Singh, and N. R. Kidwai3, "3D modified wavelet block tree coding for hyperspectral images," Indonesian Journal of Electrical Engineering and Computer Science (IJEECS), vol. 15, pp. 1001-1008, 2019.

[24] S. M. I. Repository. SMIR.Head.089Y.M.CT.4.000 [Online]. Available: https://www.smir.ch/Objects/4

[25] D. Library. DICOM Sample Modality: OT [Online]. Available: https://www.dicomlibrary.com/

[26] S. M. Wiki. Sample Data-Patient 1010: Brain MR [Online]. Available: https://wiki.idoimaging.com/index.php?title=Sample_Data

[27] S. M. Wiki. Sample Data-Patient 1030: Knee MR [Online]. Available: https://wiki.idoimaging.com/index.php?title=Sample_Data 